\title{
UNA APROXIMACIÓN AL APRENDIZAJE AUTORREGULADO EN ALUMNOS DE EDUCACIÓN SECUNDARIA
}

\author{
Fermín Torrano \\ María Soria \\ Universidad Internacional de La Rioja
}

RESUMEN: En las últimas décadas el estudio de las interrelaciones entre cognición y motivación (recogido bajo el constructo de aprendizaje autorregulado o Self-Regulated Learning) se ha convertido en tema central de la Psicología de la Educación y en una de las principales direcciones por donde avanza la disciplina. Dentro de este marco de referencia, el objetivo de la presente investigación es analizar las interrelaciones entre la motivación (metas académicas, creencias de autoeficacia y estrategias self-handicapping), las estrategias de aprendizaje y el rendimiento académico en alumnos de Educación Secundaria. La muestra estuvo compuesta por $\mathrm{N}=374$ alumnos de $2^{\circ}$ curso de la ESO (edad media 13-14 años). Los resultados ponen de relieve que estos alumnos manifiestan una alta motivación intrínseca y una elevada autoconfianza en sus capacidades académicas, además de emplear un gran número de estrategias de aprendizaje, lo cual correlaciona positivamente con el rendimiento académico. Sin embargo, las bajas puntuaciones en algunas de las escalas de estrategias cognitivas, plantea la duda de hasta qué punto se promueve el aprendizaje significativo y autorregulado en este nivel educativo. Finalmente, se proponen una serie de recomendaciones y principios para la promoción del aprendizaje autorregulado en el aula.

PALABRAS CLAVE: Psicología de la Educación, aprendizaje autorregulado, motivación académica, estrategias de aprendizaje, rendimiento académico.

\section{AN APPROACH TO THE SELF-REGULATED LEARNING IN STUDENTS OF SECONDARY EDUCATION}

\footnotetext{
ABSTRACT: In the last decades, research about self-regulated learning has become a major topic of educational psychology, and one of the essential axes of this discipline. In this context, the aim of this paper is to analyze the interrelations between motivation (academic goals, self-efficacy beliefs, and self-handicapping
} 
strategies), learning strategies and academic achievement of Secondary School students. The sample is formed by $\mathrm{N}=374$ students $13-14$ years old. The results show positive relations between all the constructs. However, the low scores in some of the scales of cognitive strategies raises the question of to what extent promotes meaningful and self-regulated learning in educational settings. Finally, we propose some guidelines for the promotion of self-regulated Learning.

KEYWORDS: Educational Psychology, self-regulated learning, academic motivation, learning strategies, academic achievement.

Recibido: 30/06/2015

Aceptado: 8/09/2015

Correspondencia: Fermín Torrano, Departamento de Ciencias del Comportamiento, Facultad de Ciencias Jurídicas, Sociales y Humanidades, Gran Vía Rey Juan Carlos I 41, 26002 Logroño (La Rioja). Email: fermin.torrano@unir.net.

\section{INTRODUCCIÓN}

El proceso de autorregulación, estudiado inicialmente desde la cibernética (Wiener, 1948), fue abordado desde una perspectiva educativa por el psicólogo americano Bandura en su libro Social Learning Theory (1977), quien lo concibió como un logro del proceso de socialización y como la capacidad para controlar las propias acciones. Según Bandura, el individuo aprende a ejecutar una serie de comportamientos que observa en otros (modelado), y que a su vez pueden estar reforzados por consecuencias externas, pero poco a poco es capaz de llevar a cabo esas conductas sin necesidad de que exista tal modelo o refuerzo.

Los posteriores estudios de Bandura sobre las creencias de los sujetos y la estructura de los sistemas autorreguladores (p. ej., Bandura, 1978, 1986) constituyen la base sobre la que se ha desarrollado una gran parte del campo de estudio sobre la autorregulación, que continúa en creciente expansión, tanto en contextos de aprendizaje-enseñanza (Karabenick y Zusho, 2015; Panadero y Alonso-Tapia, 2014; Shih, Chen, Chang y Kao, 2010; Winne, 2014), como en aquellos en los que se insiste en la importancia del control y la regulación personal, como por ejemplo, la Educación Especial (Wehmeyer et al., 2012), la Psicología Social (Baumeister, 2014), la Neuropsicología (Kelley, Wagner y Heatherton, 2015) y la Psicología del trabajo (Gagne y Deci, 2005).

En el ámbito de la Psicología de la Educación cabe reseñar la integración de las investigaciones realizadas durante los primeros años del "boom" de la revolución cognitiva (década 1970-1980), centradas en el papel de las variables cognitivas (estudios sobre el procesamiento de la información, estilos cognitivos, estrategias de aprendizaje, conocimiento previo, procesos de pensamiento, etc.) en el aprendizaje, con las realizadas en la década de 1980-1990 en el campo motivacional (estudios sobre autoconcepto, creencias de autoeficacia, atribuciones, metas, etc.), lo que dio lugar al desarrollo de un nuevo enfoque en la investigación recogido bajo el constructo de aprendizaje autorregulado (Zimmerman y Schunk, 2011). 
Hasta entonces, las tradiciones dentro de estas dos líneas de estudio habían seguido caminos muy distintos, sin tener en cuenta las posibles interconexiones que podían darse entre ellas. Por un lado, la investigación cognitiva había puesto de relieve que la diferencia entre alumnos eficaces (expertos) e ineficaces (novatos) nacía de la forma en que utilizaban las estrategias relacionadas con la adquisición, almacenamiento y recuperación de la información. Pero estos estudios, centrados en los procesos cognitivos que son necesarios para optimizar el aprendizaje, hacían poca referencia a las variables motivacionales. Como han señalado Pintrich, Roeser y DeGroot (1994), mantenían una concepción del aprendiz como "sujeto motivacionalmente inerte". Por otro lado, los estudios realizados en el campo de la motivación académica no trataban apenas la conexión entre motivación y cognición: mantenían una concepción del aprendiz como "sujeto vacío cognitivamente". Sin embargo, a partir de 1980-1990, comienzan a confluir las líneas de investigación de estos dos campos, incrementándose el interés por estudiar cómo las variables mencionadas se entrelazan e influyen en los resultados de aprendizaje.

La integración de estas dos líneas de investigación, como se ha señalado, ha dado lugar a la aparición de nuevos modelos y concepciones del aprendizaje (p. ej., aprendizaje autorregulado, aprendizaje autodirigido, etc.), basadas en la visión de los alumnos como promotores activos de su aprendizaje y de su rendimiento, en cuanto son capaces de regular su procesamiento de la información, sus creencias motivacionales y su comportamiento en orden a lograr las metas deseadas. Básicamente, estos modelos, entre los que destacan el de Pintrich (Torrano y González-Torres, 2004) y Zimmerman (Panadero y Alonso-Tapia, 2014), ambos elaborados desde la perspectiva sociocognitiva de Bandura, analizan los distintos procesos cognitivos, motivacionales/ afectivos, comportamentales y contextuales que promueven el aprendizaje, explican las relaciones que se establecen entre dichos componentes y relacionan directamente el aprendizaje con la motivación, es decir, con las metas, las atribuciones, las percepciones de competencia, la voluntad y las emociones (afectos) de los estudiantes.

\section{El aprendizaje autorregulado}

El logro de un aprendizaje autorregulado necesita tanto "voluntad" (will) como "destreza" (skill) (Torrano y González-Torres, 2004), y por esto, la educación debe ayudar a los alumnos a ser conscientes de su pensamiento, a ser estratégicos y a dirigir su motivación hacia metas valiosas. El objetivo es que los alumnos aprendan a ser sus propios maestros; y en este sentido se habla de la necesidad de pasar de la enseñanza a la práctica autorreflexiva (Schunk y Zimmerman, 2007).

De acuerdo con Zimmerman (2008), lo que caracteriza a los estudiantes autorregulados es su participación activa en el aprendizaje desde el punto de vista metacognitivo, motivacional y comportamental. Las características que se les atribuye a las personas autorreguladoras coinciden con las atribuidas a los alumnos de alto rendimiento y de alta capacidad, frente a los de bajo rendimiento o con dificultades en el aprendizaje, que presentan déficit en esas variables (Sastre-Riba, 2011; Stoeger, Fleischmann y Obergriesser, 2015; Veenham, Wilhelm y Beishuizen, 2004).

Los estudios realizados en todos los ciclos educativos coinciden en que la intervención en estrategias de aprendizaje favorece el rendimiento académico, el aprendi- 
zaje cognitivo y la motivación por aprender, y por otra, que la mejora de las creencias motivacionales de los estudiantes no sólo influye en la motivación para aprender, sino también en el modo y en la calidad con que los alumnos procesan la información, seleccionando y usando determinadas estrategias de aprendizaje (Azevedo y Hadwin, 2005; Cleary y Zimmerman, 2004; Cooper y Corpus, 2009; Dignath y Büttner, 2008; Ellis, Denton y Bond, 2014; Kistner et al., 2010; Lehmann, Hähnlein y Ifenthaler, 2014; Meece, Anderman y Anderman, 2006; Núñez et al., 2011; Perry y Winne, 2006; Puzziferro, 2008; Shih et al., 2010; Sitzmann y Ely, 2011; Taub, Azevedo, Bouchet y Khosravifar, 2014; Usher y Pajares, 2008; Winne, 2014; Winne y Jamieson-Noel, 2003; Zimmerman, 2011).

En concreto, estos estudios destacan la importancia de las "creencias de autoeficacia" (Bandura, 1977) (convicción que tiene el individuo de poder realizar con éxito la conducta que se requiere para producir unos resultados determinados) y de las "metas" académicas (razones o motivos para comprometerse en las tareas) como aspectos críticos de la motivación, que influyen en el control y regulación del aprendizaje. A este respecto, son destacables las investigaciones realizadas por Pintrich y colaboradores con el MSLQ (Motivated Strategies Learning Questionnaire) (Linnenbrink y Pintrich, 2000; Pintrich, 2003; Pintrich, Wolters y Baxter, 2000), con muestras de alumnos de secundaria y de universidad, y los estudios de Pajares, Schunk y Zimmerman (p. ej., Pajares y Schunk, 2006; Schunk y Zimmerman, 2007; Zimmerman y Schunk, 2011), en los que se destaca el positivo efecto de las creencias de autoeficacia académica en la autorregulación del aprendizaje y el rendimiento académico. Asimismo, los estudios realizados por Midgley y colaboradores en el campo de las metas académicas con el PALS (Patterns of Adaptative Learning Scales) ponen de manifiesto que las metas de aprendizaje están relacionadas de modo positivo con el uso de estrategias de aprendizaje y el rendimiento académico (Anderman, Urdan y Roeser, 2003; Midgley, 2002; Midgley et al., 2000).

Numerosas investigaciones posteriores han confirmado la influencia de estas variables motivacionales en el rendimiento académico (Anderman y Wolters, 2006; Bong y Skaalvik, 2003; de la Fuente, 2004; Lodewyk Winne Jamieson-Noel, 2009; Meece et al., 2006; Pajares y Schunk, 2001, 2006; Valentine, DuBois y Cooper, 2004; Valle et al., 2009; Wigfield y Cambria, 2010). Además de evidenciar que están relacionadas de forma positiva con el rendimiento, muestran que median los efectos de otros factores, como las capacidades académicas, el uso de estrategias, el esfuerzo y persistencia, y el rendimiento previo, en el rendimiento académico posterior. Así, se ha señalado que los mismos alumnos, con el mismo rendimiento previo y con las mismas capacidades académicas, pueden diferir en su rendimiento académico posterior a causa de las diferentes creencias motivacionales que hayan desarrollado. En concreto, la investigación muestra que las correlaciones entre autoeficacia académica y rendimiento se sitúan entre el intervalo .49 y .70, cuando tales constructoras se operativizan en nivel óptimos de especificidad y correspondencia (Pajares y Schunk, 2006). En cuanto a las metas de aprendizaje, la investigación ha determinado que su relación con el rendimiento fluctúa entre moderada y alta, y que están positivamente relacionadas con el uso de estrategias cognitivas y metacognitivas y negativamente con el uso de estrategias superficiales (Linnenbrink, 2005; Maehr y Zusho, 2009; Valle et al., 2009; Wolters, 2004). 


\section{Objetivos e hipótesis}

Bajo este marco de referencia, el objetivo de este estudio es analizar el tipo de motivación y las estrategias de aprendizaje que emplean habitualmente los estudiantes de $2^{\circ}$ curso de Educación Secundaria Obigatoria (ESO), así como la interrelación entre estos constructos y el rendimiento académico. Asimismo, y con la ayuda de los instrumentos seleccionados, nos proponemos responder a las siguientes preguntas: ¿cuáles son los puntos fuertes y débiles de los alumnos de $2^{\circ}$ curso de la ESO respecto a su motivación y al uso que hacen de las estrategias de aprendizaje?; ¿qué relaciones existen entre cada una de las variables motivacionales y de estrategias de aprendizaje y de éstas con el rendimiento académico?; ¿existen diferencias entre los alumnos de rendimiento alto, medio y bajo respecto a su motivación y a la utilización de las estrategias de aprendizaje?; y por último, ¿cuáles son las implicaciones de nuestros resultados para el trabajo de los profesores? No explicitaremos hipótesis de trabajo para cada una de las preguntas, dado que el carácter descriptivo-explicativo de nuestra investigación hace que no sea necesario (cfr. Fox, 1981; Tourón, 1989).

\section{Método}

\section{Participantes}

A través de un muestreo aleatorio por conglomerados, se seleccionaron 374 alumnos (51\% chicos y 49\% chicas), repartidos en un total de 10 aulas en centros privados y 6 aulas en centros públicos. Esta muestra constituye el 16,5\% de la población de los estudiantes matriculados en $2^{\circ}$ curso de la ESO (edad media de los sujetos: 13-14 años) en los centros de educación secundaria de Pamplona y su comarca en el momento de realizarse el estudio. El hecho de centrarnos en este nivel educativo fue debido a que muchos de estos alumnos, debido a su nivel de desarrollo cognitivo, ya pueden controlar sus procesos de aprendizaje, siendo capaces, además, de someterlos a examen. Por otro lado, consideramos que es el momento propicio para abordar de una manera sistemática la enseñanza de los procesos de autorregulación del aprendizaje.

\section{Instrumentos}

Para evaluar la motivación, se empleó el PALS, desarrollado en los años 90 y refinado a lo largo de la siguiente década por Carol Midgley y su equipo de investigación de la School of Education, de la Universidad de Michigan, con objeto de examinar las relaciones entre el ambiente de aprendizaje, la motivación, el afecto y el comportamiento de los estudiantes. (Midgley et al., 2000). Es un instrumento que está bien fundamentado teóricamente; concretamente, en la teoría de metas académicas y en las investigaciones de Carol Ames (1992). En el presente estudio nos hemos basado en la versión publicada en el año 2000, y en concreto, la versión adaptada y traducida a nuestro contexto por González-Torres y Torrano (2013). Se utilizaron las siguientes escalas:

a) Orientación a metas académicas (Personal Achievement Goal Orientations): la orientación a metas se refiere a la percepción por parte del estudiante de las razones por las que se implica en el aprendizaje. Esta dimensión engloba 2 subesca- 
las: 1) Orientación a metas de aprendizaje (Mastery Goal Orientation), que hace referencia al grado en el que el alumno se implica en el aprendizaje por razones tales como el reto, el dominio o el desarrollo de capacidades; 2) Orientación a metas de rendimiento, que a su vez se divide en otras dos, que se refiere al grado en el que el estudiante participa en el aprendizaje por el deseo de demostrar competencia (Performance-Approach Goal Orientation) o con el fin de evitar demostrar la propia incompetencia (Performance-Avoid Goal Orientation).

b) Autoeficacia académica: que comprende las percepciones de competencia que desarrollan los estudiantes a la hora de realizar las tareas académicas.

c) Uso de estrategias self-handicapping: que se refiere a las estrategias, excusas, pretextos, que utilizan algunos estudiantes para justificarse ante un mal resultado o un bajo rendimiento académico, y que de esta manera el fracaso pueda ser atribuido a esas mismas causas y no a la falta de capacidad.

Para evaluar el uso de estrategias por parte de los alumnos, se utilizó la versión del MSLQ (Motivated Strategies for Learning Questionnaire) validada en nuestro contexto por Roces, Tourón y González-Torres (1995), denominada CEAM II (Cuestionario de Estrategias de Aprendizaje y Motivación). Dado que en nuestro estudio resultaba de interés estudiar las estrategias de aprendizaje, solamente se ha utilizado la parte cognitiva del cuestionario $y$, en concreto, las siguientes escalas:

a) Repetición: refleja la utilización por parte del estudiante del recitado literal para el recuerdo de los contenidos de los libros y apuntes o para recordar palabras claves o puntos importantes.

b) Elaboración: se refiere a la construcción de conexiones entre los conocimientos a aprender, búsqueda de relaciones entre diferentes fuentes de información y de éstas con los conocimientos previos, aplicación de los conocimientos y realización de resúmenes.

c) Organización: representa la utilización por parte del alumno de recursos gráficos que le ayuden a una mejor estructuración del material de estudio y a la diferenciación entre lo importante y lo secundario.

d) Autorregulación metacognitiva: se refiere a la planificación, control y regulación del aprendizaje. La planificación comprende el establecimiento de metas y el análisis de la tarea; el control hace referencia al mantenimiento de la atención mientras se lee, a la formulación de preguntas y a la autoevaluación; y la regulación se refiere al ajuste continuo de las propias actividades cognitivas.

e) Regulación/Control del esfuerzo: se refiere a la habilidad para controlar el esfuerzo y la atención, evitar las distracciones, y regular la perseverancia en el trabajo, aunque al alumno no le guste la materia, la considere poco interesante o demasiado difícil y/o se sienta aburrido o perezoso.

\section{Procedimiento}

A los alumnos se les informó en el momento (o el día) anterior a la aplicación de los cuestionarios, y el profesor (o, en algunos casos, el tutor) estuvo presente durante el principio de la aplicación. A los alumnos se les explicó el objetivo de la investigación, la for- 
ma de responder a los cuestionarios (individualmente) y que su participación no influía en ninguna nota, siendo anónima para garantizar la confidencialidad de los resultados.

El rendimiento académico se obtuvo del siguiente modo. Los centros nos facilitaron una lista con las calificaciones de los alumnos en la evaluación final de matemáticas. Estas notas varían entre 0 (insuficiente) y 10 (sobresaliente). El resto de variables se corresponden con las respuestas dadas a los distintos ítems de los cuestionarios, cuya puntuación varía entre 1 y 7.

En el cuestionario original PALS los alumnos deben contestar individualmente con arreglo a una escala tipo Likert de 5 puntos ( $1=$ en absoluto cierto; $3=$ en algunas ocasiones cierto; $5=$ muy cierto). Sin embargo, como se empleó junto con las escalas del CEAM II, cuyo formato de respuesta es de 7 puntos, se creyó conveniente cambiar ligeramente la forma de puntuación de las escalas del PALS, pasando de una escala de 5 puntos a una de 7 ( $1=$ no, nunca; $3=$ más bien no, alguna vez; $5=$ más bien sí, con bastante frecuencia; $7=$ sí, siempre).

\section{Análisis de datos}

En primer lugar, se llevó a cabo un estudio descriptivo de las escalas, atendiendo a sus valores medios, los que permitirá conocer cuáles son los puntos fuertes y débiles de los alumnos respecto a su motivación y a las estrategias de aprendizaje que utilizan. Por lo que respecta a la relación entre las distintas variables, se calculó el coeficiente de correlación r de Pearson. Finalmente, para analizar las diferencias entre los grupos de rendimiento respecto a las variables, se llevaron a cabo análisis de varianza (ANOVA). Todos los análisis se realizaron con el programa SPSS versión 19 (Norusis, 2011). Aunque desde el punto de vista metodológico estos análisis son elementales, sin embargo, por la naturaleza de nuestro estudio se consideraron unas buenas herramientas para aproximarnos a la autorregulación que llevan a cabo los estudiantes en este nivel educativo.

\section{Resultados}

La primera de nuestras preguntas hacía referencia a ¿cuáles son los puntos fuertes y débiles de los alumnos de $2^{\circ}$ curso de la ESO respecto a su motivación y al uso que hacen de las estrategias de aprendizaje?

Respecto a la motivación (ver tabla 1), las escalas metas aprendizaje y autoeficacia tienen las medias más elevadas, mientras que la escala motivacional con la media más baja es la de estrategias self-handicapping.

Tabla 1. Valores medios de las escalas de motivación del PALS

\begin{tabular}{lc}
\hline \multicolumn{1}{c}{ ESCALA } & VALOR MEDIO \\
\hline Metas aprendizaje & 5.39 \\
Metas rendimiento (approach) & 2.74 \\
Metas rendimiento (avoidance) & 3.47 \\
Autoeficacia & 5.03 \\
Estrategias self-handicapping & 2.21 \\
\hline
\end{tabular}


En cuanto a las estrategias, las más empleadas por los alumnos son control del esfuerzo, autorregulación y repetición, mientras que las menos son elaboración y organización (tabla 2).

Tabla 2. Valores medios de las escalas de estrategias del CEAM II

\begin{tabular}{lc}
\hline ESCALA & VALOR MEDIO \\
\hline Repetición & 4.25 \\
Elaboración & 3.66 \\
Organización & 3.57 \\
Autorregulación & 4.34 \\
Control esfuerzo & 4.78 \\
\hline
\end{tabular}

En segundo lugar, se intentó dar respuesta a las posibles interrelaciones que existen entre cada una de las variables motivacionales y de estrategias de aprendizaje y de éstas con el rendimiento académico. Para ello, se calcularon los coeficientes de correlación de las diferentes variables de los cuestionarios con el rendimiento académico. Asimismo, se ha calculado la puntuación total en estrategias y se ha correlacionado con cada factor y con el rendimiento.

Tabla 31. Correlaciones de las escalas de motivación y estrategias entre sí y con el rendimiento académico

\begin{tabular}{ccccccccccccc}
\hline & $\mathbf{1}$ & $\mathbf{2}$ & $\mathbf{3}$ & $\mathbf{4}$ & $\mathbf{5}$ & $\mathbf{6}$ & $\mathbf{7}$ & $\mathbf{8}$ & $\mathbf{9}$ & $\mathbf{1 0}$ & $\mathbf{1 1}$ & $\mathbf{1 2}$ \\
\hline $\mathbf{1}$ & - &. & & & & & & & & & & \\
$\mathbf{2}$ & $.13^{*}$ & - & & & & & & & & & \\
$\mathbf{3}$ & $.11^{*}$ & $.80^{* *}$ & - & & & & & & & & \\
$\mathbf{4}$ & $.44^{* *}$ & $.16^{* *}$ & $.11^{*}$ & - & & & & & & & \\
$\mathbf{5}$ & $-.34^{* *}$ & $.26^{* *}$ & $.18^{* *}$ & $-.20^{* *}$ & - & & & & & & \\
$\mathbf{6}$ & $.39^{* *}$ & $.22^{* *}$ & $.23^{* *}$ & $.28^{* *}$ & -.10 & - & & & & & \\
$\mathbf{7}$ & $.46^{* *}$ & $.24^{* *}$ & $.25^{* *}$ & $.32^{* *}$ & $-.12^{*}$ & $.64^{* *}$ & - & & & & \\
$\mathbf{8}$ & $.34^{* *}$ & $.111^{*}$ & .08 & $.19^{* *}$ & $-.10^{*}$ & $.61^{* *}$ & $.67^{* *}$ & - & & & \\
$\mathbf{9}$ & $.63^{* *}$ & .11 & .07 & $.42^{* *}$ & $-.33^{* *}$ & $.68^{* *}$ & $.70^{* *}$ & $.62^{* *}$ & - & & \\
$\mathbf{1 0}$ & $.55^{* *}$ & -.01 & -.02 & $.41^{* *}$ & $-.50^{* *}$ & $.49^{* *}$ & $.50^{* *}$ & $.44^{* *}$ & $.71^{* *}$ & - & & \\
$\mathbf{1 1}$ & $.60^{* *}$ & $.14^{* *}$ & $.13^{*}$ & $.39^{* *}$ & $-.29^{* *}$ & $.81^{* *}$ & $.85^{* *}$ & $.79^{* *}$ & $.93^{* *}$ & $.75^{* *}$ & - & - \\
$\mathbf{1 2}$ & $.33^{* *}$ & -.07 & -.07 & $.43^{* *}$ & $-.40^{* *}$ & $.14^{*}$ & $.17^{* *}$ & $.11^{*}$ & $.34^{* *}$ & $.43^{* *}$ & $.30^{* *}$ & - \\
\hline
\end{tabular}

NOTA: se indica con un asterisco los valores significativos al nivel de 0.5 y con dos asteriscos los significativos al nivel de 0.1 .

Respecto a la motivación, las variables de autoeficacia académica y de metas de aprendizaje son las que presentan una mayor correlación con el rendimiento académico (.43 y .33 respectivamente). El resto de variables presentan una correlación nula o negativa con el rendimiento, destacando el valor negativo que alcanza la variable de estrategias self-handicapping (-.40).

1. $1=$ Metas aprendizaje; $2=$ Metas rendimiento (approach); 3= Metas rendimiento (avoidance); 4= Autoeficacia; 5= Estrategias self-handicapping; $6=$ Repetición; 7= Elaboración; 8= Organización; 9= Autorregulación; $10=$ Control esfuerzo; 11 = Total estrategias; $12=$ Rendimiento final en matemáticas. 
Las correlaciones de las metas de rendimiento (en el caso de los dos componentes approach-avoidance) con el rendimiento académico tienen un valor bajo y signo negativo en nuestro estudio. Respecto al componente de evitación (avoidance), su correlación con el rendimiento es negativa, del orden de -.04 a -.19. Por otro lado, en el caso del componente de aproximación (approach), no encontramos evidencia empírica para afirmar que su correlación con el rendimiento sea positiva; sin embargo, los resultados evidencian que están relacionadas con el uso de estrategias cognitivas.

Finalmente, la correlación de las estrategias self-handicapping con el rendimiento, así como con otras muchas variables motivacionales (p. ej., metas de aprendizaje) y de estrategias (p. ej., autorregulación, control del esfuerzo), es negativa y considerable (-.40 o mayor).

Las correlaciones de las variables correspondientes a las estrategias de aprendizaje y el rendimiento académico son ligeramente más altas que las de la motivación. La correlación más alta corresponde al factor control del esfuerzo (.43), seguida del factor autorregulación (.34) y de la puntuación total de estrategias (.30). De las estrategias cognitivas (cuya correlación con el rendimiento, en el caso de que englobáramos todos los ítems referidos a tales estrategias en una única escala, es de .16 y significativa al nivel de .01), las que tienen una mayor correlación con el rendimiento son las de elaboración (.17), seguidas, al nivel de .01, de las estrategias de repetición (.14) y de organización (.11).

Para analizar las diferencias entre los grupos de rendimiento respecto a las variables motivacionales y de estrategias se llevaron a cabo varios análisis de varianza. Para ello, dividimos a los alumnos en tres grupos de rendimiento: grupo 1, de rendimiento bajo, formado por los alumnos con unas notas entre el 0 y el 4 (insuficiente); grupo 2, de rendimiento medio, formado por los alumnos con unas notas entre el 5 (suficiente) y el 6 (bien); y grupo 3, de rendimiento alto, formado por los alumnos con unas notas entre el 7 (notable) y el 10 (sobresaliente). Pretendemos saber si los alumnos, en función de sus resultados académicos, valoran de modo significativamente distinto cada una de las variables de los cuestionarios.

En la tabla 4 se recogen los resultados correspondientes al análisis de varianza de las escalas motivacionales.

Como se puede ver a través del valor del estadístico de prueba F, existen diferencias significativas entre los grupos de rendimiento en las siguientes variables motivacionales: metas aprendizaje, autoeficacia y estrategias self-handicapping. Estas diferencias son significativas al nivel de .01 Los únicos aspectos en los que se producen diferencias significativas entre todos los grupos entre sí (1-2, 1-3 y 2-3), como revela el índice de Bonferroni, son la autoeficacia y las estrategias self-handicapping. En la variable de metas aprendizaje sólo se presentan diferencias entre los grupos 1-2 y 1-3, mientras que en el resto no existen diferencias entre grupos.

Para todos las variables en las que se da una significación entre las valoraciones de los grupos ha resultado significativo, al nivel de .01, el componente lineal (c. lineal), de lo que se deduce no sólo la existencia de una relación entre las variables, sino también que la función que mejor se ajusta a esa relación sería la de una recta. A mayor rendimiento se produce una mejor valoración de las variables del cuestionario de motivación (en el caso de las metas y de las creencias de autoeficacia). 
Tabla 4. Análisis de varianza de las escalas motivacionales con el rendimiento

\begin{tabular}{|c|c|c|c|c|c|c|c|}
\hline \multirow[b]{2}{*}{ VARIABLE } & \multicolumn{5}{|c|}{$\begin{array}{l}\text { MEDIA GRUPOS DE } \\
\text { RENDIMIENTO } \\
\end{array}$} & \multirow[b]{2}{*}{$\begin{array}{l}\text { ANÁLISIS } \\
\text { TENDENCIA }\end{array}$} & \multirow[b]{2}{*}{$\begin{array}{c}\text { COMPARACIONES } \\
\text { MÚLTIPLES }\end{array}$} \\
\hline & $\begin{array}{l}\text { MEDIA } \\
\text { TOTAL }\end{array}$ & BAJO & MEDIO & ALTO & $F$ & & \\
\hline Metas aprendizaje & 5.33 & 4.82 & 5.34 & 5.68 & $11.35^{* *}$ & c. lineal** & $1-2,1-3$ \\
\hline $\begin{array}{l}\text { Metas rendimiento } \\
\quad \text { (approach) }\end{array}$ & 2.72 & 2.89 & 2.73 & 2.59 & 1.05 & & \\
\hline $\begin{array}{l}\text { Metas rendimiento } \\
\text { (avoidance) }\end{array}$ & 3.40 & 3.50 & 3.57 & 3.13 & 2.47 & & \\
\hline Autoeficacia & 4.98 & 4.31 & 4.92 & 5.57 & $26.83^{* *}$ & c. lineal** & $1-2,1-3,2-3$ \\
\hline Self-handicapping & 2.19 & 2.68 & 2.20 & 1.81 & $21.87^{* *}$ & c. lineal** & $1-2,1-3,2-3$ \\
\hline
\end{tabular}

NOTA: se indica con un asterisco los valores significativos al nivel de 0.5 y con dos los significativos al nivel de 0.1 .

En la tabla 5 se presentan los resultados correspondientes al análisis de varianza de las escalas de estrategias de aprendizaje. Al igual que antes, en dicha tabla, los valores significativos al nivel de .05 están representados con un asterisco y al nivel de .01 con dos asteriscos.

Tabla 5. Análisis de varianza de las escalas de estrategias con el rendimiento

\begin{tabular}{|c|c|c|c|c|c|c|c|}
\hline \multirow[b]{2}{*}{ VARIABLE } & \multicolumn{5}{|c|}{$\begin{array}{l}\text { MEDIA GRUPOS DE } \\
\text { RENDIMIENTO }\end{array}$} & \multirow[b]{2}{*}{$\begin{array}{c}\text { ANÁLISIS } \\
\text { TENDENCIA }\end{array}$} & \multirow[b]{2}{*}{$\begin{array}{c}\text { COMPARACIONES } \\
\text { MÚLTIPLES }\end{array}$} \\
\hline & $\begin{array}{l}\text { MEDIA } \\
\text { TOTAL }\end{array}$ & BAJO & MEDIO & ALTO & $F$ & & \\
\hline Repetición & 4.22 & 3.79 & 4.49 & 4.24 & $6.91^{* *}$ & c. lineal* & $1-2$ \\
\hline Elaboración & 3.64 & 3.28 & 3.80 & 3.74 & $5.75^{* *}$ & c. lineal* & $1-2,1-3$ \\
\hline Organización & 3.55 & 3.25 & 3.68 & 3.61 & 2.43 & & \\
\hline Autorregulación & 4.31 & 3.80 & 4.39 & 4.61 & $17.48^{* *}$ & c. lineal** & $1-2,1-3$ \\
\hline Control esfuerzo & 4.77 & 4.07 & 4.82 & 5.27 & $24.48^{* *}$ & c. lineal** & $1-2,1-3,2-3$ \\
\hline Total estrategias & 4.13 & 3.66 & 4.26 & 4.35 & $12.91 * *$ & c. lineal** & $1-2,1-3$ \\
\hline
\end{tabular}

NOTA: se indica con un asterisco los valores significativos al nivel de 0.5 y con dos los significativos al nivel de 0.1 .

Como se puede ver, existen diferencias significativas al nivel de .01 entre los grupos de rendimiento en todas las variables de estrategias consideradas, excepto en el caso de las estrategias de organización, en la que todos los alumnos, a pesar de su rendimiento, tienden a puntuar de forma muy similar. El único aspecto en el que se producen diferencias significativas entre todos los grupos (1-2, 1-3 y 2-3) es el control del esfuerzo. En las escalas de elaboración, autorregulación y estrategias total sólo se 
presentan diferencias entre los grupos 1-2 y 1-3, mientras que en el caso de las estrategias de repetición las diferencias sólo se dan entre el primer grupo y el segundo.

Para todas las escalas en las que se da una significación entre las valoraciones de los grupos ha resultado significativo el componente lineal, con lo que se comprueba, como se ha señalado anteriormente, no sólo la existencia de una relación entre las variables, sino también que la función que mejor se ajusta a esa relación sería la de una recta. Al igual que en la parte motivacional, a mayor rendimiento se produce una mejor valoración de gran parte de las variables del cuestionario de estrategias.

\section{Conclusiones}

Como se pone de relieve, el tipo de motivación que caracteriza al alumno de $2^{\circ}$ curso de la ESO es uno de los más beneficiosos para el aprendizaje, ya que manifiesta una alta motivación intrínseca (esto es, dice implicarse en el aprendizaje por motivos relacionados con el desarrollo de sus capacidades y el dominio de la tarea) y una elevada confianza en sus capacidades académicas: cree que si estudia y se esfuerza aprenderá los contenidos y tendrá éxito en las tareas.

Las estrategias más empleadas por los alumnos de $2^{\circ}$ curso de la ESO son control del esfuerzo seguidas de las de autorregulación, repetición, elaboración y organización, lo cual nos indica que los alumnos muestran una alta voluntad y disposición para realizar el trabajo y que suelen supervisar sus procesos de pensamiento antes, durante y después de estudiar y de la realización de las diferentes tareas. Sin embargo, al mismo tiempo, observamos que no emplean (o con menos frecuencia) las estrategias cognitivas profundas (de elaboración y organización) relacionadas con el aprendizaje significativo y autorregulado.

Las bajas puntuaciones obtenidas por los alumnos en los ítems de elaboración y de organización parecen indicar que, en general, no se están consiguiendo algunos de los objetivos que se consideran prioritarios en la ESO, tales como la necesidad de ayudar al estudiante a integrar los nuevos conocimientos con aquellos que ya posee (aprendizaje significativo) y desarrollar la competencia para organizar, manejar, relacionar y evaluar la información (aprendizaje autónomo). Por el contrario, de nuestros resultados se podría deducir, más bien, que los profesores parecen dar más importancia a que el alumno aprenda lo que ya viene dado en los libros o lo que el docente transmite como un producto ya elaborado que a que construya y desarrolle su conocimiento, aportándole un sentido personal. Por otro lado, creemos que la ausencia en el empleo de estas estrategias también radica en el alumno. En concreto, nos planteamos hasta qué punto el esfuerzo que manifiestan realizar es "inteligente", es decir, está dirigido a la puesta en marcha y aplicación de tales estrategias de procesamiento profundas o, simplemente, es un esfuerzo relacionado con un aprendizaje superficial y con el uso de estrategias de bajo nivel (de repetición) limitado a cumplir con los requerimientos mínimos para realizar las tareas.

Según se desprende de los datos, se podría decir que estos alumnos están motivados para aprender y siguen con atención las clases, pero como meros espectadores. Probablemente, en ellas obtendrán unos apuntes que, en el peor de los casos, serán su única fuente de información. Estos resultados dibujan un panorama poco deseable para el aprendizaje significativo y autorregulado, en el que el estudiante ha de elaborar y cons- 
truir de forma activa sus conocimientos, aportándoles un sentido personal. Sin embargo, nos preguntamos: ¿hasta qué punto el alumno es inducido a trabajar de este modo por los profesores? ¿̇se está enseñando a los estudiantes las estrategias más importantes para analizar, seleccionar y comprender la información, y el conocimiento condicional, que ayuda a establecer cuándo, cómo y por qué una estrategia es adecuada en una situación y no lo es en otra? y ¿no podría ocurrir que las clases de matemáticas sean excesivamente expositivas, informativas, unidireccionales, y que los estudiantes sólo se limiten a tomar apuntes y a memorizarlos, sin llegar a elaborar y organizar eficazmente la información, e integrarla en sus conocimientos previos? No hay duda que el estudiante está influido por el contexto didáctico en mayor o menor grado, por lo que los profesores deberían reflexionar, a nuestro juicio, sobre estas cuestiones y analizar su estilo docente, considerando en qué medida están potenciando y promoviendo el uso de estrategias de elaboración y organización y una actividad intelectual rica en los alumnos (Butler, Schnellert y Cartier, 2013; Tourón y Santiago, 2015).

Con relación a la motivación, las variables de autoeficacia académica y metas de aprendizaje son los que presentan una mayor correlación con el rendimiento académico. El resto de variables presentan una correlación nula o negativa con el rendimiento, destacando los valores negativos que alcanza las estrategias self-handicapping. Asimismo, las creencias de autoeficacia académica y las metas de aprendizaje presentan correlaciones muy elevadas con las estrategias de aprendizaje, lo que parece indicar, como muestra la investigación, que una motivación adecuada también puede influir en el rendimiento de manera indirecta a través de su relación con la implicación cognitiva del alumno.

Las correlaciones de las metas de rendimiento (approach-avoidance) con el rendimiento académico tienen un valor bajo y signo negativo en nuestro estudio. En el caso del componente de aproximación (approach) de las metas de rendimiento, no encontramos evidencia empírica para afirmar que su correlación con el rendimiento sea positiva, tal como se muestra en algunos estudios dentro de este campo (Harackiewicz, Durik, Barron, Linnenbrink y Tauer, 2008; Muis y Winner, 2012). No obstante, los resultados también muestran que estas metas están relacionadas con el uso de estrategias cognitivas, lo que parece indicar que, en ciertas situaciones (p. ej., ante tareas difíciles o irrelevantes), dicha orientación puede conducir a los alumnos a involucrarse en las tareas, utilizando las estrategias cognitivas necesarias para lograr sus objetivos, y obtener un buen rendimiento académico.

Las correlaciones de las variables correspondientes a las estrategias de aprendizaje y el rendimiento académico son ligeramente más altas que las de la motivación. La correlación más alta corresponde a la escala control del esfuerzo, seguida de la de autorregulación y puntuación total de estrategias. De las estrategias cognitivas, las que tienen una mayor correlación con el rendimiento son las de elaboración, seguidas de las de repetición y de organización. Las correlaciones de las estrategias de control del esfuerzo y de autorregulación con el rendimiento académico ponen de manifiesto la importancia de la dimensión personal sobre los aspectos técnicos del estudio. En concreto, los factores relacionados con la voluntad del sujeto (esfuerzo, perseverancia, diligencia) son los que presentan una mayor relación con el rendimiento, mientras que los aspectos más técnicos, englobados dentro de la escala de organización, muestran una relación muy pequeña con el mismo. Cualquier inter- 
vención educativa tendrá que tener en cuenta este aspecto. No tiene sentido enseñar al alumno un conjunto de técnicas si no se logra paralelamente que desarrolle un mayor sentido de voluntad y de responsabilidad personal, que le haga implicarse en el estudio día a día, fijarse planes de estudio y cumplirlos, y aprovechar bien el tiempo que emplea en estudiar.

Finalmente, los resultados de esta investigación revelan que existen diferencias globales significativas en función del rendimiento en todas las escalas motivacionales y de estrategias a excepción de metas rendimiento (approach-avoidance) y organización. En general, a mayor rendimiento se produce una mejor valoración de las variables evaluadas. Los alumnos de rendimiento alto están más motivados y utilizan en mayor medida las estrategias de aprendizaje que los de rendimiento medio. A su vez, éstos están por encima de los alumnos de rendimiento bajo. Sólo en el caso de las estrategias cognitivas de repetición y de elaboración, los alumnos de rendimiento medio se sitúan por encima de los de rendimiento alto.

\section{Implicaciones para los profesores}

Nuestros resultados ponen de relieve la influencia de algunas variables internas al sujeto, como la motivación y las estrategias de aprendizaje, en el rendimiento académico. Por ello, las intervenciones para la mejora del aprendizaje autorregulado y del rendimiento deberían ir acompañadas de cambios en el clima del aula, en la práctica docente del profesor y en la manera en que éste enseña y evalúa su materia, de modo que:

a) La clase se convierta en una guía para el alumno y no en la única fuente de conocimiento. Los apuntes y los libros deberían dejar de ser una garantía para obtener un buen rendimiento y convertirse en facilitadores de futuros aprendizajes.

b) Se planteen actividades en el aula que, debido a su complejidad, requieran por parte de los estudiantes una regulación consciente y deliberada de su conducta de manera que para realizarlas se vean obligados a planificar previamente su actuación, controlar y supervisar lo que están haciendo y les parezca útil evaluar su ejecución cuando la concluyan.

c) Se enseñe a los alumnos los beneficios y la utilidad del empleo de las estrategias de aprendizaje y se facilite la transferencia de las estrategias utilizadas a otras materias, tareas y contextos.

d) En los exámenes se requiera al estudiante que explique, relacione, aplique y reflexione sobre lo aprendido, y no sólo que reproduzca la información transmitida por el profesor.

e) Se involucre a los alumnos en la toma de decisiones dentro del aula y se fomente el diálogo, la participación y la independencia. Asimismo, es necesario dar oportunidades al estudiante para que, progresivamente, asuma parte de la responsabilidad de su aprendizaje.

f) Se incluya la enseñanza de estrategias como uno de los contenidos esenciales de los cursos de formación de profesores. A este respecto, se requiere que el 
profesor sea con su actuación un facilitador de conocimiento y del proceso de aprendizaje más que un mero aplicador de técnicas y de propuestas de intervención sugeridas por la investigación. Estas ideas son recogidas por los nuevos modelos de formación y desarrollo del profesor, en los que se destaca la enseñanza como profesión del aprendizaje. Desde esta perspectiva, se pone de manifiesto que para enseñar al estudiante a utilizar estratégicamente sus recursos en situaciones de aprendizaje es necesario que previamente el profesor sea capaz tanto de aprender como de enseñar estratégicamente los contenidos de su materia. En concreto, se recomiendan los proyectos de investigaciónacción como camino en esta línea de formación del profesorado (Butler et al., 2013).

\section{Limitaciones y futuras direcciones}

Los hallazgos encontrados constituyen una aproximación a cómo los alumnos de $2^{\circ}$ curso de la ESO autorregulan su aprendizaje. Sin embargo, hay que ser prudentes, ya que dichos resultados están condicionados por varios hechos: a) han sido obtenidos a partir de la aplicación de cuestionarios de autoinforme; b) los análisis de correlación efectuados sólo nos permiten inferir el grado y la dirección en que las variables covarían, pero no permiten, como ya es sabido, establecer un nexo de causalidad de una variable respecto de otra; c) se ha tomado como marco de referencia la asignatura de matemáticas (lo cual exige contrastar los resultados con el resto de asignaturas del currículo); y d) se trata de un análisis global, donde se apuntan determinadas tendencias y en el que algunos alumnos y profesores pueden no sentirse aludidos o afectados por los resultados expuestos.

Además de desarrollar programas de intervención para la mejora del aprendizaje y del rendimiento que incluyan aspectos motivacionales y cognitivos, es necesario seguir elaborando y validando instrumentos de medida, que permitan diagnosticar la realidad motivacional y cognitiva de los alumnos y los avances experimentados tras la aplicación de tales programas.

Por último, la constatación del importante papel que juega la voluntad del sujeto en el proceso de aprendizaje hace necesario, además, que estos programas tengan entre sus finalidades el fortalecimiento de la voluntad, la formación de hábitos de estudio y la valoración del esfuerzo personal. En nuestra opinión, con un adecuado entrenamiento en esas dimensiones, todos los estudiantes podrán mejorar su grado de control sobre el aprendizaje y el rendimiento y paliar muchas de las dificultades de aprendizaje que presentan, particularmente, los sujetos de rendimiento bajo.

\section{REFERENCIAS BIBLIOGRÁFICAS}

Ames, C. (1992). Classrooms: Goals, structures, and student motivation. Journal of Educational Psychology, 84, 261-271. doi: 10.1037//0022-0663.84.3.261.

Anderman, E. M., Urdan, T. y Roeser, R. (2003). The Patterns of Adaptive learning Survey: History, development and psychometric properties. Comunicación presentada en Indicators of Positive Development Conference, Washington. 
Anderman E. M., y Wolters, C. (2006). Goals, values, and affects: influences on student motivation. En P. Alexander y P. Winne (Eds.), Handbook of Educational Psychology, New York: Simon y Schuster/Macmillan. doi:10.4324/9780203874790. ch17.

Azevedo, R., y Hadwin, A. F. (2005). Scaffolding self-regulated learning and metacognition- implications for the design of computer-based scaffolds. Instructional Science, 33, 367-379.

Bandura, A. (1977). Social learning theory. Englewood Cliffs, NJ: Prentice-Hall. (Trad. cast: Teoría del aprendizaje social. Madrid: Espasa Calpe, 1982).

Bandura, A. (1978). The self system in reciprocal determinism. American Psychologist, 33, 344-358.

Bandura, A. (1986). Social foundations of thought and action. Englewood Cliffs, NJ: Prentice Hall. (Trad. cast: Pensamiento y acción. Fundamentos sociales. Barcelona: Martínez Roca, 1987).

Baumeister, R. F. (2014). Self-regulation, ego depletion, and inhibition. Neuropsychologia, 65, 313-319.

Bong, M., y Skaalvik, E. (2003). Academic self-concept and self-efficacy: How different are they really? Educational Psychology Review, 15, 1-40.

Butler, D. L., Schnellert, L., y Cartier, S. C. (2013). Layers of self- and co-regulation: Teachers' co-regulating learning and practice to foster students' self-regulated learning through reading. Education Research International. doi: 10.1155/2013/845694.

Cleary, T. J.. y Zimmerman, B. J. (2004). Self-regulation empowerment program: A School-based program to enhance self-regulated and self-motivated cycles of student learning. Psychology in the Schools, 41, 537-550.

Cooper, C. A., y Corpus, J. H. (2009). Learners' developing knowledge of strategies for regulation motivation. Journal of Applied Developmental Psychology, 30, 525-536.

Dela Fuente, J. (2004). Perspectivas recientes en el estudio de la motivación: la teoría de la orientación de meta. Revista Electrónica de Investigación Psicoeducativa, 2, 35-62.

Dignath, C.. y Büttner, G. (2008). Components of fostering self-regulated learning among students. A meta-analysis on intervention studies at primary and secondary school level. Metacognition and Learning, 3, 231-264. doi: 10.1007/s11409-0089029-x

Ellis, A. K., Denton, D. W., y Bond, J. B. (2014). Analysis of research on metacognitive teaching strategies. Procedia - Social and Behavioral Sciences, 116, 4015-4024.

Fox, D. (1981). El proceso de investigación en educación. Pamplona: EUNSA.

Gagne, M., y Decir, E. L. (2005). Self-determination theory and work Motivation. Journal of Organizational Behavior, 26, 331-362.

González-Torres, M. C. y Torrano, F. (2013). Perfiles de motivación y rendimiento académico en matemáticas en estudiantes de educación secundaria: Utilidad del Patterns of Adaptive Learning Scales (PALS). En V. Mellado, L. J. Blanco, A. B. Borrachero y J. A. Cárdenas (Eds.), Las Emociones en la Enseñanza y el Aprendizaje de las Ciencias Experimentales y las Matemáticas (pp. 177-215). Badajoz: DEPROFE. 
Harackiewicz, J. M., Durik, A. M., Barron, K. E., Linnenbrink-Garcia, L., y Tauer, J. (2008). The role of achievement goals in the development of interest: Reciprocal relations between achievement goals, interest, and performance. Journal of Educational Psychology, 100, 105-122. doi: 10.1037/0022-0663.100.1.105.

Karabenick, S. A., y Zusho, A. (2015). Examining approaches to research on self-regulated Learning: conceptual and methodological considerations. Metacognition and Learning, 10, 151-163. doi: 10.1007/s11409-015-9137-3.

Kelley, W. M., Wagner, D. D., y Heatherton' T. F. (2015). In search of a human selfregulation system. Annual Review of Neuroscience, 38, 389-411. doi: 10.1146/ annurev-neuro-071013-014243.

Kistner, S., Rakoczy, K., Otto, B., Dignath-van Ewijk, C., Buttner, G., y Klieme, E. (2010). Promotion of self-regulated learning in classrooms: Investigating frequency, quality, and consequences for student performance. Metacognition and Learning, 5, 157-171. doi: 10.1007/s11409-010-9055-3.

Lehmann, T., Hähnlein, I., y Ifenthaler, D. (2014). Cognitive, metacognitive, and motivational perspectives on preflection in self-regulated online learning. Computers in Human Behavior, 32, 313-323.

Linnenbrink, E. A. (2005). The dilemma of performance-approach goals: The use of multiple goal contexts to promote students' motivation and learning. Journal of Educational Psychology, 97, 197-213.

Linnenbrink, E. A., y Pintrich, P. R. (2000). Multiple pathways to learning and achievement: The role of goal orientation in fostering adaptative motivation, affect, and cognition. En C. Sansone y J. M. Harackiewicz (Eds.), Intrinsic and Extrinsic Motivation (pp. 195-226). San Diego, CA: Academic Press.

Lodewyk, K., Winne, P. H., y Jamieson-Noel, D. L. (2009). Implications of task structure on self-regulated learning and achievement. Educational Psychology, 29, 1-25.

Maehr, M. L., y Zusho, A. (2009). Achievement goal theory: The past, present, and future. En K. R. Wentzel y A. Wigfield (Eds.), Handbook of motivation in school (pp. 77-104). New York: Taylor \& Francis.

Meece, J. L., Anderman, E. M., y Anderman, L. H. (2006). Classroom goal structures, student motivation, and academic achievement. Annual Review of Psychology, 57, 487-503. doi: 10.1146/annurev.psych.56.091103.070258.

Midgley, C., Maehr, M. L., Hruda, L. Z., Anderman, E., Anderman, L., Freeman, K. E., Gheen, M., Kaplan, A., Kumar, R., Middleton, M. J., Nelson, J., Roeser, R., y Urdan, T. (2000). Manual for the Patterns of Adaptive Learning Scales (PALS), Ann Arbor, MI: University of Michigan. Recuperado de http://www.umich.edu/ pals/ pals/PALS\%202000_V13Word97.pdf.

Midgley, C. (Ed.) (2002). Goals, goal structure and pattern of adaptive learning. Mahwah, NJ: Lawrence Erlbaum.

Muis, K. R., y Winne, P. H. (2012). Assessing the psychometric properties of the achievement goals questionnaire Across Task Contexts. Canadian Journal of Education, 35, 232-248.

Norusis, M. (2011). IBM SPSS Statistics 19: Guide to data analysis. Chicago, IL: SPSS. 
Núñez, J. C., Cerezo, R., Bernardo, A., Rosario, R., Valle, A., Fernández, E., y Suárez, N. (2011). Implementation of training programs in self-regulated learning strategies in Moodle format: Results of a experience in higher education. Psicothema, 23, 274-281.

Pajares, F., y Schunk, D. H. (2001). Self-Beliefs and School Success: Self-Efficacy, SelfConcept, and School Achievement. En R. Riding y S. G. Rayner (Eds.), International Perspectives on Individual Differences: Self-Perception (Vol. 2, pp. 239-265). Westport, CT: Ablex.

Pajares, F., y Schunk, D. H. (2006). The self and academic motivation. Theory and research after the cognitive revolution. En J. M. Royer (Ed.), The Cognitive Revolution in Educational Psychology (pp. 165-185). Greenwich, CT: Information Age.

Panadero, E., y Alonso-Tapia, J. (2014). ¿Cómo autorregulan nuestros alumnos? Revisión del modelo cíclico de Zimmerman sobre autorregulación del aprendizaje. Annales de Psicología, 30, 450-462.

Perry, N. E., y Winne, P. H. (2006). Learning from learning kits: gStudy traces of students' self-regulated engagements using software. Educational Psychology Review, 18, 211-228.

Pintrich, P. R. (2003). Motivation and classroom learning. En W. M. Reynolds y G. E. Miller (Eds.), Handbook of Psychology: Educational psychology (Vol. 7, pp. 103122). Hoboken, NJ: Wiley.

Pintrich, P. R., y Linnenbrink, E. A. (2000, April). The role of motivation in intentional learning. Comunicación presentada en el Meeting of the American Educational Research Association, New Orleans.

Pintrich, P. R., Roeser, R. W., y DeGroot, E. V. (1994). Classroom and individual differences in early adolescents' motivation and self-regulated learning. Journal of Early Adolescence, 14, 139-161.

Pintrich, P. R., Wolters, C., y Baxter, G. P. (2000). Assessing metacognition and selfregulated learning. En G. Schraw y J. C. Impara (Eds.), Issues in the Measurement of Metacognition (pp. 43-97). Lincoln: Buros Institute of Mental Measurements, University of Nebraska Press.

Puzziferro, M. (2008). Online technologies self-efficacy and self-regulated learning as predictors of final grade and satisfaction in college-level online courses. The American Journal of Distance Education, 22, 72-89.

Roces, C., Tourón, J., y González-Torres, M. C. (1995). Validación preliminar del CEAM II. Psicológica, 16, 347-366.

Sastre-Riba, S. (2011). Funcionamiento metacognitivo en niños con altas capacidad. Revista de Neurología, 52, 11-18.

Schunk, D. H., y Zimmerman, B. J. (2007). Motivation and self-regulated learning: Theory, research, and applications. Mahwah, NJ: Lawrence Erlbaum.

Shih, K., Chen, H. C., Chang, C. Y., y Kao, T. C. (2010). The development and implementation of scaffolding based self-regulated learning system for e/m-learning. Educational Technology \& Society, 13, 80-93. 
Sitzmann, T., y Ely, K. (2011). A meta-analysis of self-regulated learning in workrelated training and educational attainment: What we know and where we need to go. Psychological Bulletin, 137, 421-442. doi: 10.1037/a0022777.

Stoeger, H., Fleischmann, S., y Obergriesser, S. (2015). Self-regulated learning (SRL) and the gifted learner in primary school: The theoretical basis and empirical findings on a research program dedicated to ensuring that all students learn to regulate their own learning. Asia Pacific Education Review, 16, 257-267. doi: 10.1007/ s12564-015-9376-7.

Taub, M., Azevedo, R., Bouchet, F., y Khosravifar, B. (2014). Can the use of cognitive and metacognitive self-regulated learning strategies be predicted by learners' levels of prior knowledge in hypermedia-learning environments? $C H B, 39,356-367$.

Torrano, F., y González-Torres, M. C. (2004). El aprendizaje autorregulado: presente y futuro de la investigación. Revista Electrónica de Investigación Psicoeducativa, 2, 1-34.

Tourón, J. (1989). Métodos de estudio en la universidad. Pamplona: EUNSA.

Tourón, J., y Santiago, R. (2015). El modelo flipped learning y el desarrollo del talento en la escuela. Revista de Educación, 368, 196-231. doi: 10.4438/1988-592X0034-8082-RE.

Usher, E. L., y Pajares, F. (2008). Sources of self-efficacy in school: Critical Review of the literature and future directions. Review of Educational Research, 78, 751-796.

Valentine, J. C., DuBois, D. L., y Cooper, H. (2004). The Relation between self-beliefs and academic achievement: A Meta-Analytic Review. Educational Psychologist, 39, 111-133.

Valle, A., Rodríguez, S., Cabanach, R. G., Núñez, J. C., González-Pienda, J. A., y Rosario, P. (2009). Metas académicas: perspectiva histórica y conceptual e implicaciones educativas. Electronic Journal of Research in Educational Psychology, 7, 1073-1106.

Veenman, M. V., Wilhelm, P., y Beishuizen, J. J. (2004). The relation between inteIlectual and metacognitive skills from a developmental perspective. Learning and Instruction, 14, 89-109.

Wehmeyer, M., Shogren, K., Palmer, S., Williams-Diehm, K., Little, T., y Boulton, A. (2012). The impact of the self-determined learning model of instruction on student self-determination. Exceptional Children, 78, 135-153.

Wiener, N. (1948). Cybernetics: Control and communication in the animal and the machine. Cambridge, MA: MIT Press.

Wigfield, A., y Cambria, J. (2010). Students' achievement values, goal orientations, and interest: Definitions, development, and relations to achievement outcomes. Developmental Review, 30, 1-35.

Winne, P. H. (2014). Issues in researching self-regulated learning as patterns of events. Metacognition and Learning, 9, 229-237. doi: 10.1007/s11409-014-9113-3.

Winne, P. H., y Jamieson-Noel, D. (2003). Self-regulating studying by objectives for learning: Students' reports compared to a model. Contemporary Educational Psychology, 28, 259-276. 
Wolters, C. (2004). Advancing achievement goal theory: Using goal structures and goal orientations to predict students' motivation, cognition, and achievement. Journal of Educational Psychologist, 96, 236-250.

Zimmerman, B. J. (2008). Investigating self-regulation and motivation: Historical background, methodological developments, and future prospects. American Educational Research Journal, 45, 166-183.

Zimmerman, B. J. (2011). Motivational sources and outcomes of self-regulated learning and performance. En B. J. Zimmerman y D. H. Schunk (Eds.), Handbook of selfregulation of learning and performance (pp. 49-64). New York: Routledge.

Zimmerman, B. J., y Schunk, D. H. (2011). Handbook of self-regulation of learning and performance. New York: Routledge. 
